\title{
OBE教育理念下应用化学专业综合实验课程的创新实践
}

\author{
刘珊珊 ${ }^{*}$, 韩艳阳*, 冯凯, 崔伟, 刘义, 张震, 翁永根, 何涛, 李庆忠 \\ 烟台大学化学化工学院, 山东烟台 264005
}

\begin{abstract}
摘要: 针对应用化学综合实验课程内容陈旧、教学模式不利于学生创新能力的培养, 及其自主学习能力发挥、教学评价 方式单一等问题, 基于 OBE (Outcome-based education)教育理念对该课程进行了系统的教学设计: 采取将科研成果与 教学内容相融合的方式设计创新型实验项目, 提高实验内容的前沿性、创新性及科学性; 课堂教学增加设计性、探索性 及挑战性环节, 激发学生的学习兴趣及自主学习意识, 培养学生的创新能力及科学精神; 建立以成果为导向的教学评价 模式, 强化过程考核, 完成课程目标达成度评价。根据课程目标达成度评价结果及持续改进的原则优化教学方案, 推动 实验教学向能力导向培养模式发展。
\end{abstract}

关键词: OBE理念; 化学实验; 创新实践

中图分类号: G64; O6

\section{Innovative Practice of Comprehensive Experiment Course of Applied Chemistry under OBE Concept}

\author{
Shanshan Liu *, Yanyang Han *, Kai Feng, Wei Cui, Yi Liu, Zhen Zhang, Yonggen Weng, Tao He, \\ Qingzhong Li \\ College of Chemistry and Chemical Engineering, Yantai University, Yantai 264005, Shandong Province, China.
}

\begin{abstract}
Owing to the outdated content and one-sided assessment method in the comprehensive experimental course of applied chemistry, the development of students' innovative ability and independent learning is limited. In this article, the course is systematically designed based on the OBE (Outcome-based education) concept to solve these problems. Firstly, the innovative experimental projects are designed by integrating teachers' achievements in scientific research. Hence, the students will experience the frontier and innovative technology in a scientific way. Secondly, some design, exploring and challenging projects are carried out during teaching and learning process to stimulate students' learning interest and self-learning awareness. And through these projects, students' innovative ability and scientific spirit are cultivated. Thirdly, the process assessment is highlighted, and the outcome-oriented assessment mode is as well as established to evaluate the achievement of course objectives. Eventually, the teaching scheme is optimized and expected to promote the development of the ability-oriented training mode in experimental course by applying the evaluation results of the course objectives achievement and the continuous improvement principle.
\end{abstract}

Key Words: OBE concept; Chemical experiment; Innovative practice

收稿: 2021-07-25; 录用: 2021-07-28; 网络发表: 2021-09-02

“通讯作者, Emails: liushanshan@ytu.edu.cn (刘珊珊); yyhan1026@163.com (韩艳阳)

基金资助: 烟台大学教学改革研究项目(jyxm2021015, jyxm2020015) 


\section{1 引言}

Outcome-Based Education (OBE) 即成果导向教育, 是一种以学生为中心 ${ }^{[1]}$, 以成果产出为导向 ${ }^{[2]}$ 的先进教育理念, 要求将学习产出、教学活动、目标达成度评价有机统一起来, 教学活动以学习目 标与成果为导向 ${ }^{[3]}$, 由传统的以 “教” 为中心向以 “学” 为中心转变, 更大限度地激发学生的学习 积极性和主观能动性, 培养学生的创新意识和专业应用能力。以OBE理念为导向进行专业课程改革, 有助于推动专业毕业要求的达成, 进而实现本科人才培养目标。烟台大学应用化学专业以培养高素 质应用型化学人才为目标, 要求本专业学生不但具有扎实的理科化学功底, 而且具有利用所学化学 知识、思想、实验技能等发现、分析和解决问题的能力。本文基于OBE教育理念, 以学生为中心、成 果产出为导向、持续改进为原则对应用化学专业综合实验课程进行创新性改革实践, 提高了实验教 学质量以及学生对所学化学知识的应用能力, 推动了实验教学向能力导向培养模式发展。

\section{2 烟台大学 “应用化学专业综合实验”课程情况简介}

烟台大学应用化学专业紧密围绕山东省高端化学、化工产业布局和新旧动能转换战略的重大需 求, 聚焦精细化学品开发、化工新材料、贵金属资源利用三大核心领域, 对标国家一流专业标准, 培养掌握化学基础理论知识和技能, 具有良好道德情操、职业素养和团队协作精神, 具备较强专业 竞争能力、实践创新能力及终身学习能力, 能够在化学化工、材料、能源等领域从事科学研究、化 学品开发、质量控制、生产管理和服务的高素质应用型专业人才。应用化学综合实验课程是为应用 化学专业大三学生开设的专业实验课程, 共64学时。该实验课程旨在通过化学化工、材料、能源等 方向相关的综合实验培养学生分析、解决问题的综合能力及对所学化学知识的应用能力, 进而为应 用化学专业人才培养目标的达成提供支撑, 课程目标详见表 1 。

表1 应用化学综合实验的课程目标

\begin{tabular}{|c|c|}
\hline 课程目标 & 课程目标要求 \\
\hline 课程目标1 & $\begin{array}{l}\text { 能综合应用化学基本原理和化工基本原理, 借助文献研究, 分析实验过程中存在的影响因素, 并对实 } \\
\text { 验结果获得有效结论 }\end{array}$ \\
\hline 课程目标2 & 在实验方案设计操作中能够考虑社会、健康、安全及环境等因素的约束 \\
\hline 课程目标3 & $\begin{array}{l}\text { 掌握常见无机有机纳米材料等化合物的实验合成、分析、鉴定和测试方法, 能够搭建实验装置, 并采 } \\
\text { 用科学的实验方法安全地开展实验 }\end{array}$ \\
\hline 课程目标4 & $\begin{array}{l}\text { 能根据文献及相关的要求查阅文献, 独立设计实验方案, 并进行操作。能合理采集及整理实验数据, } \\
\text { 具备基于专业知识对实验结果进行分析和解释的能力, 并获得合理有效的结论 }\end{array}$ \\
\hline 课程目标5 & $\begin{array}{l}\text { 培养学生具有良好的人际交往能力, 能独立完成团队分配的任务, 具有良好的团队组织、协调能力, } \\
\text { 具备团队合作精神 }\end{array}$ \\
\hline 课程目标6 & 能够认识不断学习的必要性, 具有自主学习和终身学习的能力 \\
\hline
\end{tabular}

然而, 目前该实验课程在课程内容、教学方式以及考核方式等方面均与基础实验课区别不大, 依然侧重加强学生对基础理论知识的理解和基本实验技能的掌握, 导致课程目标无法完全支撑毕业 要求的达成。具体问题如下: (1) 课程内容方面: 部分课程内容陈旧(如表2所示)、实验仪器和方法 老旧, 未做到与时俱进, 不利于学生综合实验技能、创新能力及化学素养的培养; (2) 课堂教学方 面: 传统的教学模式没有在培养学习兴趣和创新能力的重要环节给予学生充分的自由度, 存在 “削 足适履” 的现象, 限制了学生创新能力的发挥, 教学环节枯燥且缺乏挑战性, 不利于课程目标4、5、6 的达成; (3) 教学评价方面: 目前该课程的考核评价途径和方式单一, 与成果导向的达成度评价方式 不相符, 难以实现教学质量的持续改进。 
大学 化 学 Univ. Chem. 2021, 36(11), 2107075 (3 of 5)

表2 应用化学专业综合实验课程内容

\begin{tabular}{|c|c|c|}
\hline 实验项目 & 实验内容 & 实验目标 \\
\hline 贝壳中多种成分分析 & $\begin{array}{l}\text { 测定若干种贝壳中主要成分 (钻, 镁、有 } \\
\text { 机物等)和微量成分 (铁, 铅, 钾等)的含量 }\end{array}$ & $\begin{array}{l}\text { 分小组合作, 完成从样品制备到结果处理全过程。主要内容 } \\
\text { 是常量分析和分光光法操作。用马弗炉高温灼烧测定有机物 } \\
\text { 含量 }\end{array}$ \\
\hline 桔皮中有效成分提取 & 桔皮中果胶提取及测定 & 学习采用常见的提取方法和超声波辅助提取方法 \\
\hline 三氯化六氨合钴制备及其分 & 有无机原料合成配位化合物, 并测定其 & 要求掌握配位化合物相关知识, 及沉淀、酸碱、氧化还原三 \\
\hline 子式确定、结构表征 & 组成 & 大滴定, 磁矩测定, 成单电子数判断, 分离能测定及计算 \\
\hline 以锌焙砂为原料制备超细氧 & 无机纳米材料的制备 & 学习纳米材料的制备方法, 了解纳米结构特性, 化妆品的配 \\
\hline 化锌及氧化锌的表征和应用 & 熟悉精细化工 & 制 \\
\hline 中药丹参滴丸的制备 & 利用物理化学手段制备中药滴丸 & 了解滴丸制备原理, 学习传统中药滴丸的制作方法 \\
\hline $\begin{array}{l}\text { 六氧合铁酸钾的制备及磁化 } \\
\text { 率的测定 }\end{array}$ & 配合物制备, 磁化率的测定原理与方法 & 无机制备及磁天平测磁化率的原理 \\
\hline
\end{tabular}

\section{3 基于OBE理念的创新实践}

针对应用化学专业综合实验课程存在的问题, 基于OBE理念, 遵循成果导向的反向设计、以学 生为中心、持续改进三个关键原则, 对课程内容、教学模式和教学考核评价进行了综合改革。

\section{1 课程内容更新}

根据2020版应用化学综合实验的课程目标(表1)持续更新实验内容。近年来, 烟台大学化学化工 学院引进了很多高学历青年教师, 其科研背景涵盖新能源、新材料、3D打印、纳米材料、生物医药、 环境治理等高新科技领域, 并且拥有领先的科研成果。基于此, 我们开展了将科研成果转化为实验 教学项目的探索。具体而言, 以课程目标为导向, 采用 “科研反哺教学” 的策略, 与时俱进地增设 能支撑课程目标的新型实验项目 (表3), 提高实验内容的创新性、新颖性及科学性, 融入先进的化学 合成方法、结构与性能表征技术和仪器分析方法, 培养学生的创新能力及科学精神, 并提升其分析 问题、解决问题的能力, 最终达成课程目标要求。

表3 应用化学综合实验新型实验项目设计

\begin{tabular}{|c|c|c|}
\hline 课程目标 & 实验项目 & 科研方向 \\
\hline $1,3,4,5$ & 纳米二氧化钛光催化剂的制备、活性评价及活性与缺陷关系的综合实验 ${ }^{[4]}$ & 光催化 \\
\hline $1,3,4,5$ & 氢氧化镍薄膜制备及析氧电催化活性评价的综合实验 ${ }^{[5]}$ & 电催化 \\
\hline $3,4,5,6$ & $\mathrm{KH}_{2} \mathrm{PO}_{4}(\mathrm{KDP})$ 多晶原料合成、单晶生长及其性能表征的综合实验设计 & 晶体生长 \\
\hline $1,2,3,4,5,6$ & Fabricating Flexible Packaging Batteries in General Chemistry Laboratories ${ }^{[6]}$ & 锂离子电池 \\
\hline $2,3,4,6$ & 铜离子荧光探针罗丹明酰肼的合成及性能检测 & 重金属检测 \\
\hline $1,2,3,4,5$ & 丁香酚的分离及香兰素的合成 & 有机合成 \\
\hline $3,4,5,6$ & 多色彩苂光树脂制备及3D打印 & 3D打印 \\
\hline
\end{tabular}

\section{2 教学模式设计及教学过程评价}

创新教学模式, 遵循以学生为中心的原则, 注重 “量体裁衣”, 避免 “削足适履”。摒弃验证性 的基础实验教学方法, 增加具有设计性、探索性及挑战性的教学环节, 进而激发学生的学习兴趣, 增强学生的学习主动性和创新意识。教学过程遵循成果导向的反向设计原则, 根据应用化学综合实 验课程目标, 设计课前预习报告、文献调研、科技前沿介绍、实验方案设计、思考题、课堂互动、 
小组讨论、实验数据分析及报告、课程论文等体现成果产出的环节(表4), 课前指导教师只给出实验 要解决的问题, 学生分组自主进行文献调研、实验原理、内容、步骤等环节的设计, 提出实验方案, 在与指导教师讨论后独立开展实验操作, 完成数据处理与分析讨论; 增设实验项目数量, 给予学生 自由选择的机会, 最大限度地发掘学生的兴趣点及创新思维。另一方面, 成果导向的教学评价是 $\mathrm{OBE}$ 理念的关键组成部分之一, 这就要求指导教师对表4中能够体现成果产出的环节进行过程评价, 将一 锤定音式评价转变为分阶段、分环节评价, 并制定相应的评分标准, 最终实现目标达成度评价。

表4 落实课程目标的教学模式及环节

\begin{tabular}{cl}
\hline 课程目标 & \multicolumn{1}{c}{ 教学模式及环节 } \\
\hline 课程目标 1 & 课前预习报告, 文献调研, 课堂互动, 实验数据分析, 实验报告 \\
课程目标 2 & 文献调研, 案例教学, 科技前沿介绍, 实验方案设计 \\
课程目标 3 & 实验方案设计, 实验操作 \\
课程目标 4 & 文献调研, 实验方案设计, 课堂互动, 思考题, 实验数据分析, 实验报告 \\
课程目标 5 & 分组实验, 小组讨论, 实验方案设计, 实验操作, 课堂互动 \\
课程目标 6 & 科技前沿介绍, 案例教学, 文献调研, 课前预习报告, 思考题, 课堂互动 \\
\hline
\end{tabular}

以 “软包锂离子电池的制备及性能表征” 实验为例：(1) 在预习环节, 指导教师设置促进课程目 标达成的课前思考题并提供部分参考文献, 由每组学生调研锂离子电池种类及研究现状、正负极材 料种类以及软包锂离子电池的组装工艺及性能表征方法等问题, 通过文献调研完成课前思考题并提 交文献总结报告, 以PPT形式汇报调研结果, 指导教师根据文献总结报告和答辩情况进行评价。(2) 实 验方案设计环节, 各实验小组在预习基础上, 充分讨论设计出实验方案, 指导教师根据实验方案的 合理性、严谨性、可行性、环保性、安全性等给予评价, 并对学生的实验方案给出指导性意见。(3) 实 验操作环节, 主要是对学生的态度、操作规范性、条理性等进行评价, 此环节同样可设置具有课程 目标导向的思考题及课堂互动环节, 如引导学生思考电池性能测试过程中充电电容大于放电电容的 原因: $38 \%$ 的学生认为是由于电池各部件之间的接触不良, $20 \%$ 的学生认为电池内部漏电减少了放电 电容, $42 \%$ 的学生认为充电过程中发生了不可逆的副反应。对于前两种回答, 学生可以通过实验自 行验证排除。指导教师根据学生作答情况和互动表现进行评价, 该互动环节可促进课程目标 $1 、 4 、 6$ 的达成。(4) 数据处理与结果分析环节, 学生提交实验报告, 指导教师对数据处理的正确性、特别是 实验结果分析讨论以及改进方案的深度、逻辑性进行打分。另外, 基于本课程实验学生可以在教师 指导下撰写科研论文, 投稿录用的可获得加分项。

\section{3 具体成效及持续改进措施}

目前 6 个新型实验项目 “纳米二氧化钛光催化剂的制备、活性评价及活性与缺陷关系的综合实验 设计” “氢氧化镍薄膜制备及析氧电催化活性评价的综合实验设计” “铜离子荧光探针罗丹明酰肼的 合成及性能检测” “软包锂离子电池的制备及性能表征” “丁香酚的分离及香兰素的合成” 及 “ $\mathrm{KH}_{2} \mathrm{PO}_{4}(\mathrm{KDP})$ 多晶原料合成、单晶生长及其性能表征的综合实验设计” 正式投入了教学, 实验过 程中学生表现出浓厚的学习兴趣, 更加体现了寓教于乐, 提升了创新意识和专业应用能力。相关的 研究成果已整理成教研论文, 其中 2 篇已发表于核心期刊《化学教育》上, 1 篇已被该期刊录用; 另 有一篇教研论文已发表于美国化学会(ACS)的Journal of Chemical Education期刊。在此基础上, 根据 OBE理念持续改进的原则及教学评价效果, 不断优化实验教学方案的同时, 设计更多切实可行的创 新型实验项目, 以满足多样化的学生兴趣。为了提高应用化学综合实验的高阶性、落实以学生为中 心的教学理念, 本实验课将进行如下的教学改革尝试: 对当前实验教学项目在实验内容、课堂设计以 及过程评价等方面进行进一步优化, 着力打造 8 个精品实验项目, 并且将实验项目学时由 8 学时增加到 32 学时, 在这 8 个实验项目中学生凭兴趣选做两个实验项目, 推动实验教学向能力导向培养模式发展。 


\section{4 结语}

基于OBE理念对应用化学专业综合实验进行改革, 采用 “科研反哺教学” 策略使学生在课堂上 即可接触与专业相关的某个科研或应用课题, 进而不断发掘学生的专业兴趣, 促使他们用心钻研, 避 免以往实验教学过程中的 “蜻蜓点水” “走马观花” 的形式主义。在教学过程中秉承以学生为中心的 原则, 根据教学效果反馈对课程体系持续改进, 以保持应用化学综合实验课程体系的创新性、高阶 性、挑战度, 这不但有利于激发学生学习热情, 而且有利于学生创新能力、综合实验能力及科学精 神的培养。

\section{参 考 文 献}

[1] 李志义. 中国高等教育, 2014, No. 21, 19.

[2] 李志义. 中国高等教育, 2014, No. 17, 7 .

[3] 张树永. 大学化学, 2019, 34 (11), 4.

[4] 何涛, 王立博, 王海花, 赵玉华, 翁永根, 郭清华, 于瑞晓, 黄浩. 化学教育, 2017, 38 (18), 44.

[5] 何涛, 李高鹏, 刘浩因, 王海花, 赵玉华, 谢晓清. 化学教育, 2020, 41 (2), 104.

[6] Liu, S. S.; Ge, X. L.; Zhang, T.; Han, Y. Y.; Cui, W.; Guan, R. G.; Yang, X.; He, T.; Li, Q. Z.; Feng, K.; et al. J. Chem. Educ. $2021,98,2471$. 